\title{
Safety state identification of concrete pumping pipeline based on multi-channel audio signals
}

\author{
Guanghui $\mathrm{He}^{1, *}$ \\ ${ }^{1}$ Shanghai Construction Group Co., Ltd, Shanghai 201114, China
}

\begin{abstract}
Based on the principle of superposition and attenuation of sound propagation, a Multi-point Multi-channel noise Reduction Method (MMRM) for audio monitoring is proposed. With the help of the proposed noise reduction method, the sounds made by the concrete pumping-pipelines themselves on the construction site and the environmental noises are separated in real time. Then, the Mel-Frequency Cepstral Coefficients (MFCCs) and spectral centroids of the filtered pumping-pipeline signals are extracted in real time as the features. Finally, the distance between the currently obtained feature vector of the pipeline signals and the previous accumulated MFCC clustering are determined as the parameter for safety identification. In order to verify the actual effects of the MMRM and the analysis accuracy of MFCCs in the clustering, several numerical tests and field measurements are carried out. The numerical results show that the MMRM has an excellent performance on noise reduction; the feature vectors composed of the MFCCs with length no greater than 4 are sufficient for the recognition and clustering of pumping-pipeline operation state.
\end{abstract}

\section{Introduction}

Concrete pumping technology is widely used in the construction of high and huge structures in civil engineering, such as casting of high-rise concrete buildings and construction of concrete pylon for long span bridge. However, in the process of concrete pumping, the pumping pipes are frequently blocked due to the improper concrete composition or incorrect operation of equipment. At present, the safety state assessment and early warning of pumping pipes has become one of the most urgent problems in the concrete construction.

In order to deeply understand the principle of the blockage of pumping pipes, the pumping process of concrete has been studied from the perspective of mechanics. Secrieru et al [1] studied the flow of concrete in the pumping pipes by field test and numerical simulation. It was found that the blockage of the pumping pipes was mainly caused by the excessive pressure of flowing concrete. And this excessive pressure was caused by the excessive frictional resistance of concrete lubrication layer inside the pipe-friction resistance on the inner wall of the steel pipe. The stress assessment and prediction techniques were then developed according to the findings. Ngo et al [2] carried out an experimental study and proposed a prediction model to formulate the relationships among concrete

\footnotetext{
* Corresponding author: flamehe@163.com
}

grading, pumping rate, concrete-pump wall friction and concrete pressure. Wu et al [3] studied the influence of flow rate and water-cement ratio of high strength concrete on pressure loss in pumping pipes and they obtained the relevant calculation method. Kwon et al [4] reviewed the current research status of concrete pumping pipes and flowing concrete interaction system in detail and they also concluded the current measuring methods of the properties of pumped concrete lubricating layers and the research progress of the prediction model of rheological concrete pumping parameter.

Using the research results of mechanical analysis of concrete pumping, it is possible to monitor the pressure state of flowing concrete in pumping-pipelines. Kaplan et al [5] and Feys et al [6] optimized the empirical formula, which related the frictional stress and the pressure loss of the inner wall of the pipelines, and the friction stress and pressure of the lubricating layer of flowing concrete were monitored indirectly by a tribometer placed on the inner wall of pipelines. Chen et al [7] obtained and verified the empirical relationship between the surface strain of the pipeline and the pressure on the inner wall of the pipeline, and they also monitored the flowing concrete pressure on the inner wall of the pipeline by measuring the strain on the outer surface of the pipeline. With the help of the database which recording the interfacial yielding stress inside the pipeline, viscosity constants and concrete grading, Mai et al [8] established a computational model relating the concrete grading to yielding stress and pressure inside 
the pipeline. This model was able to evaluate the pressure inside the pipeline with relative error around $15 \%$.

Being different from previous safety monitoring, which relied on the mechanical analysis of rheological concrete-pump-pipe interaction system, this study attempts to monitor the status of pipelines using the technology of sound signal recognition. Sound recognition technology is widely used in fields, such as the concrete damage diagnosis and monitoring [9, 10], concrete strength detection [11], mechanical fault diagnosis [12] and liquid pipes leakage detection [13]. Compared with the pickup of parameters like frictional stress of flowing concrete, strain of pipeline and velocity measurement of concrete, the pickup of sound of pipelines is easy and will do no damage to the pipelines. Moreover, it shows no disadvantage of easy wear and short life of the sensor. In order to improve the clarity of sound signal acquisition of pipelines, this paper proposes a multi-point and multi-channel noise reduction algorithm based on the superposition principle of sound propagation and energy attenuation principle. It adopts Mel-Frequency Cepstral Coefficients (MFCC) [14] and spectrum centroids [15] as sound features to perform sound state clustering analysis [16]. In the framework of the algorithm, the current status of pipeline can be judged with the help of historical sound and the currently collected samples.

\section{Principle of Sound Recognition System}

For new concrete pumping facilities, the failure sound signal characteristics are unknown, that is, the clustering label of the pumping sound is unknown. Therefore, the supervised machine learning method cannot be used. In this paper, an unsupervised learning method [16] is adopted to conduct the cluster analysis on the short-time spectrum centroids and MFCC features of sound.

Figure 1 shows the flow chart of sound classification for pumping pipes. Due to the random and high-decibel construction noise, the noise is appropriately filtered by multi-point and multi-channel noise reduction method to obtain the sound signals with high signal-to-noise ratio. Assuming that the pipeline works normally at the beginning (usually several minutes, $T_{0}$ ) of sound acquisition, the sound segment can be divided and windowed into frames, and then the feature coefficients, which are the short-time spectrum centroids and short-time MFCC vectors, of each frame can be extracted as the "normal" cluster. Meanwhile, the convex-hull [17] (the outer boundary of feature points) of the feature points are computed. Then, the short-time feature coefficients of each frame corresponding to the audio signal after time $T_{0}$ are extracted, and the distance $d$ between the new audio frame feature coefficients and the "normal" convex hull is calculated frame by frame. Obviously, $d \leq 0$ means that the newly collected frames belong to the "normal" category while $d>0$ means that the newly collected frames may belong to the "normal" category or the "abnormal" category. Therefore, the distance $\alpha$ greater than 0 is introduced as the threshold. When $d>\alpha$, the classification algorithm initially determines that the sample is "abnormal", at the same time, the pipeline safety administrator will come to check whether the system is misjudged. If the system is misjudged by manual check, the threshold value $\alpha$ of the system should be increased properly. Otherwise, the "abnormal" feature set should add new members. After a long time of audio sampling and clustering analysis, the system can modify more reliable threshold and "abnormal" feature set. The number of clusters will be increased and each category will have labels such as "normal", "slightly blocked", "moderately blocked" and "severely blocked". The process of "deterioration" of pumping-pipelines is usually the process of the increase of parameter $d$, so the system takes this parameter as the early warning parameter of pipelines.

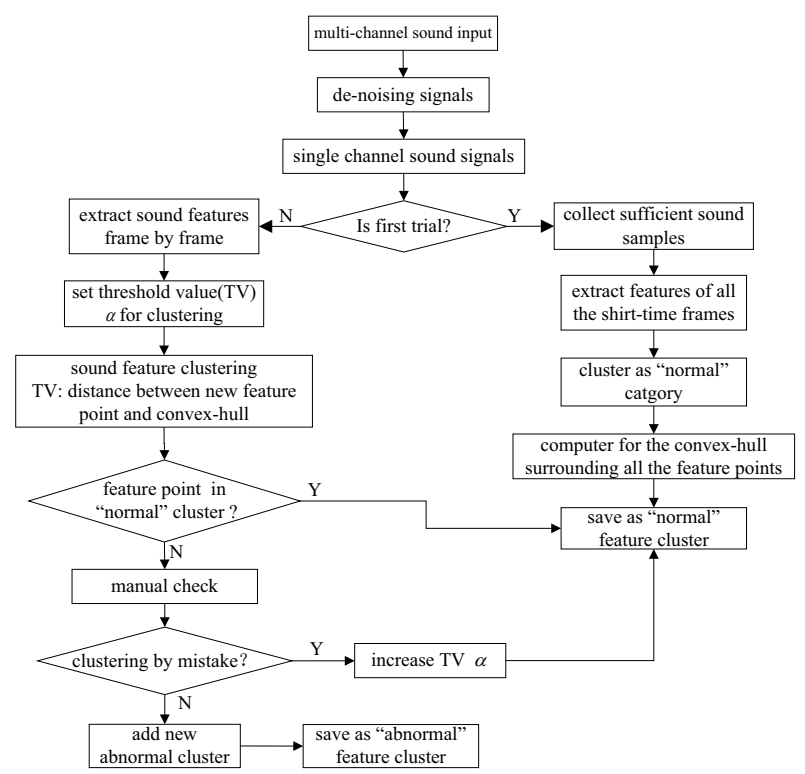

Fig. 1. Flow chart of sound classification for pumping pipe

\section{Signal De-noising Method}

The sound of pipelines collected in construction site is often accompanied by high-decibel environmental random noise. Effective noise reduction is one of the keys to correct sound recognition and classification before the application of sound recognition algorithms.

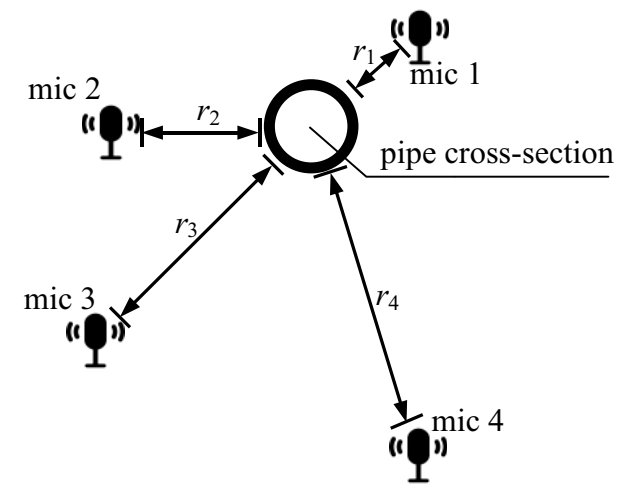

Fig. 2. Diagram of sound recording devices around the pipeline 
In this paper, the method shown in Figure 2 is used to pick up sound signals. Four recording devices, mic 1, mic 2 , mic 3 and mic 4 , are arranged on the same plane with distances $r_{1}, r_{2}, r_{3}$ and $r_{4}$ away from the pipeline, respectively. The acoustic amplitude signals are synchronously picked up at the same sampling rate SR, which are $\mathbf{B}_{1}=\left\{B_{1 i}\right\}, \mathbf{B}_{2}=\left\{B_{2 i}\right\}, \mathbf{B}_{3}=\left\{B_{3 i}\right\}$ and $\mathbf{B}_{4}=\left\{B_{4 i}\right\}, i$ $=1,2, \ldots, n$.

It is assumed that the ambient noise propagates from a distance away from the pipeline and the ambient noise is approximately a parallel sound wave. The ambient noise is approximately the same everywhere in the range of the four recording devices. Moreover, acoustic amplitude time series $\mathbf{E}=\left\{E_{i}\right\}, i=1,2, \ldots, n$ is adopted. The sound of the pipeline is approximately assumed to be a point source and the intensity of the vibration signal received by the recording device is inversely proportional to the distance between the pipeline and the recording device. Based on the above assumption, it can be concluded that

$$
\begin{gathered}
\mathbf{B}_{j}=\mathbf{R}_{j}+\mathbf{E}, \quad j=1,2,3,4 . \\
\mathbf{R}_{j}=\left\{\frac{k_{i}}{\delta_{i}+r_{j}} R_{i}\right\}, \quad i=1,2, \cdots, n . j=1,2,3,4 .
\end{gathered}
$$

Among them, $\left\{R_{i}\right\}$ is the amplitude time series of the sound wave excited by the pipeline itself around the pipeline; $\left\{k_{i}\right\}$ and $\left\{\delta_{i}\right\}$ are the unknown environmentrelated interference time series.

Applying the discrete fourier transform to equation (1), yields that

$$
\mathbf{F}_{\mathrm{B} j}=\mathbf{F}_{R j}+\mathbf{F}_{E}, \quad j=1,2,3,4 .
$$

where, $\mathbf{F}_{B j}=\operatorname{dft}\left(\mathbf{B}_{j}\right)$ represents the discrete fourier transform of time series $\mathbf{B}_{j} ; \quad \mathbf{F}_{R j}=\operatorname{dft}\left(\mathbf{R}_{j}\right)$ represents the discrete fourier transform of time series $\mathbf{R}_{j}$; $\mathbf{F}_{E}=\operatorname{dft}(\mathbf{E})$ represents the discrete fourier transform of time series $\mathbf{E}$; dft denotes discrete fourier transform, and the fast fourier transform algorithm is used in this paper.

In the actual process of audio acquisition, the audio recording device, mic 1 , is usually placed around the pipeline. Therefore, the value of $r_{1}$ is set as zero in the paper, as a result,

$$
\mathbf{R}_{1}=\left\{R_{i}\right\}, \quad i=1,2, \cdots, n .
$$

The four relations provided by the equation (3) form the algebraic equations with respect to unknowns: $\left\{k_{i}\right\}$, $\left\{\delta_{i}\right\},\left\{E_{i}\right\}$ and $\left\{R_{i}\right\}$. And the solution of $\mathbf{F}_{\mathrm{R} 1}$ can be obtained as

$$
\mathbf{F}_{\mathrm{R} 1}=\frac{\left(\mathbf{a}_{1}\left(r_{2}-r_{4}\right)+\mathbf{a}_{2}\left(r_{3}-r_{2}\right)+r_{4}-r_{3}\right)\left(\mathbf{F}_{B 1}-\mathbf{F}_{B 2}\right)}{\mathbf{a}_{2}\left(r_{4}-r_{2}\right)+\mathbf{a}_{1}\left(r_{2}+r_{3}\left(\mathbf{a}_{2}-1\right)-\mathbf{a}_{2} r_{4}\right)}(5)
$$

where sequences $\mathbf{a}_{1}$ and $\mathbf{a}_{2}$ are defined as follows:

$$
\mathbf{a}_{1}=\frac{\mathbf{F}_{B 1}-\mathbf{F}_{B 2}}{\mathbf{F}_{B 1}-\mathbf{F}_{B 3}}, \quad \mathbf{a}_{2}=\frac{\mathbf{F}_{B 1}-\mathbf{F}_{B 2}}{\mathbf{F}_{B 1}-\mathbf{F}_{B 4}}
$$

Equation (5) can be inverted to a time series of wave amplitude around the pipeline by inverse discrete fourier transform as

$$
\mathbf{R}=\operatorname{idft}\left(\mathbf{F}_{\mathrm{R} 1}\right)
$$

in which, idft represents the inverse discrete fourier transform, and the fast inverse discrete fourier transform algorithm is adopted. Obviously, the inverted sequence $\mathbf{R}$ uses the sound signals picked up by multiple channels to eliminate the noise signal $\mathbf{E}$. The accuracy of sound signal obtained by equation (7) mainly depends on the non-parallel characteristics of noise in the environment and the sensitivity of acoustic reflection interference in complex construction environment mentioned in the assumption (1).

\section{Sound Signature Parameters}

Each frame of sound wave amplitude records picked up by recording device is composed of huge number of data points, e.g. for a sound frame whose length is $30 \mathrm{~ms}$, and its sampling rate is $44.1 \mathrm{kHz}$, the number of data points reach 1323 per frame. Therefore, it is inefficient and unreliable [14] to characterize wave sequences directly with time series in clustering analysis of sound signals. In this paper, the spectral centroids and MFCC of each frame are used to characterize the features of sound.

\subsection{Spectral Centroids}

For a continuous stationary sound sample $\mathbf{x}=\left\{x_{i}\right\}, i=$ $1,2, \ldots n$, a discrete fourier transform coefficient $\mathbf{X}=\left\{X_{i}\right\}, i=1,2, \ldots n$ and spectral centroid $C$ can be defined as

$$
C=\frac{s \sum_{m=1}^{\lceil n / 2\rceil} m\left|X_{m}\right|^{2}}{n \sum_{m=1}^{\lceil n / 2\rceil}\left|X_{m}\right|^{2}}
$$

In the equation, || denotes modular operation of complex numbers and \lceil\rceil represents the up-rounding of a real number. $s$ represents the audio sampling rate: the number of waveform points collected per second.

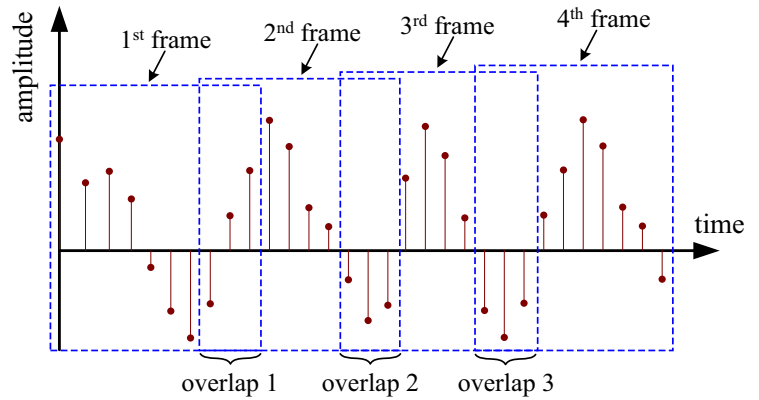

Fig. 3. Four successive frames with length 10 and overlap 3

The operation sound of pipeline and the construction site environmental sound are statistically non-stationary, which makes the fourier transform unavailable. In order to avoid this problem, and make available the algorithms sucn as discreat fourier transform, the continuous sound signals are windowed into frames with same length, and the spectral centroids of each frame are computed. As is shown in Figure 3, the acoustic amplitude signals are divided into a series of successive frames with each containing an approximately stationary sample of number $n$. In addition, in order to smooth the short-time 
fourier transform transition, there are some overlapping areas between adjacent frames, abd appropriate window function correction is considered to weaken the signal on both sides of each frame. In this paper, the short time series is modified by Hanming [14] window function $W_{i}$ , and the modified sequence is

$$
\tilde{x}_{i}=W_{i} x_{i}
$$

where,

$$
W_{i}=\frac{25}{46}-\frac{21}{46} \cos \left(\frac{2 \pi(i-1)}{n-1}\right), i=1,2, \cdots, n .
$$

As a measure of the average frequency of spectrum, spectral centroids are often used to describe the brightness of sound. Generally speaking, low and deep sound tends to have a lower spectral centroid while clear and sharp sound tends to have a higher spectral centroid. This kind of characteristic has been applied in the sound signal in the spectrum stable environment, such as the underwater sound signal recognition [18]. For a construction site with complex sound sources, as is shown in Figure 4, the wall decoration friction sound, rock drill operation sound and the sound in the elevator compartment under vertical starting condition are collected from a construction site, and the short-time spectrum centroids of them are calculated with $50 \mathrm{~ms}$ as the Hanming window width and $25 \mathrm{~ms}$ as the overlapping width of adjacent windows. As can be seen in the figure, there is a certain proportion of overlapping area of spectral centroids between the elevator and the rock drill. In addition, the frictional sound is sharper than the former (the high-frequency component is dominant) and its spectral centroid can be distinguished significantly from the other two. Therefore, in this paper, the spectral centroid is considered as a component of the acoustic feature vector but not as a whole.

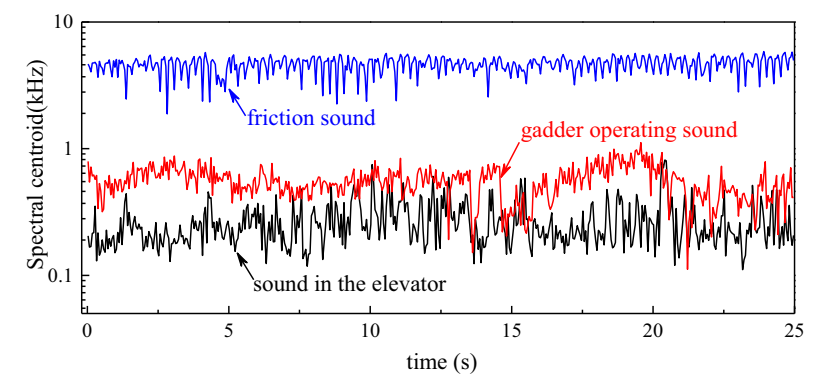

Fig. 4. Spectral centroids of different sound signals

\subsection{Mel-Frequency Cepstral Coefficients}

When the short-time spectrum centroid is used as the recognition feature of sound, it is inevitable that there will be insufficient discrimination of the noises in construction site and pipeline. So, in this paper, Mel Frequency Cepstral Coefficient (MFCC) is introduced to supplement the feature vector of short time sound signal.

The MFCC as a classification feature, has been widely used in sound classification and recognition and has become one of the research hotspots. The calculation of MFCC is also based on fourier analysis, so the framing, the overlapping of adjacent frames and the windowing function shown in Figure 3 are also applicable to the calculation of MFCC. For the short-time signal sequence $\tilde{\mathbf{x}}=\left\{\tilde{x}_{i}\right\}, i=1,2, \ldots, n$ with the window in each frame, the main calculation steps of MFCC consist of the following steps:

1) Short-time fourier transform. After the fast fourier transform, the discrete fourier transform coefficient $\tilde{\mathbf{X}}=\operatorname{fft}(\tilde{\mathbf{x}})$ is obtained, the $m$ th coefficient corresponds to the frequency $m s / n$, where $s$ is the sample rate.

2) Design of triangular band-pass filter. As is shown in Figure 5, the recorder bank consists of $L$ triangular recorders, the $i$ th central frequency of the filter is $f_{i}, i=1$, $2, \ldots, L$. The spacing of central frequencies increases with the number of filters, however, the corresponding Mel-Frequencies

$$
f_{\text {mel } i}=2595 \log _{10}\left(1+f_{i} / 700\right)
$$

are in equidistant distribution, the spacing is

$$
f_{\text {gap }}=\frac{f_{\text {mel max }}-f_{\text {mel min }}}{L+1}
$$

where, $f_{\text {melmax }}$ and $f_{\text {melmin }}$ represent maximum and minimum Mel-Frequencies, respectively. The minimum possible value of $f_{\text {melmin }}$ is zero; the maximum possible value of $f_{\text {melmax }}$ is $2595 \log _{10}(1+s / 1400)$.

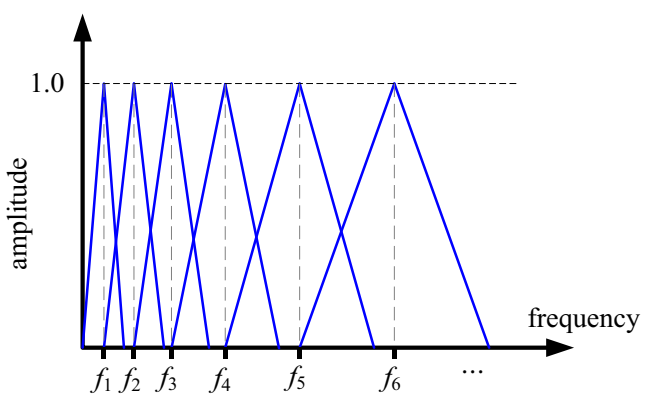

Fig. 5. Schematic diagram of a triangular bandpass filter bank

3) Calculation of the weighted average of triangular bandpass filter of $\tilde{\mathbf{X}}$.

$$
Y\left(m_{i}\right)=\sum_{m=1}^{n} \log \left|\tilde{X}_{i}\right| H_{i}\left(\frac{m}{n} s\right), i=1,2, \cdots n .
$$

where, the weight $H_{i}(\bullet)$ is determined by the $i$ th band-pass filter. The weight should be zero when the value of $m s / n$ falls outside the triangle.

4) Generation of the MFCCs. First, the filter bank sequence is defined as

$$
\mathbf{Y}=Y(m)=\left\{\begin{array}{l}
Y\left(m_{i}\right), \quad m=m_{i} \\
0, \quad \text { otherwise }
\end{array}\right.
$$

then, the inverse discrete cosine transform is applied to $\mathbf{Y}$ to obtain the MFCC

$$
\mathbf{c}_{\text {mel }}=\operatorname{idct}(\mathbf{Y})
$$

in which, the idct represents inverse discrete cosine transform. Obviously, the length of $\mathbf{c}_{\mathrm{mel}}$ is $n$. In the application of sound classification and recognition, only the first few components of MFCC vector are taken to fully characterize the sound features.

Based on the three sound samples used in the analysis in section 4.1, Figure 6 shows the first three-dimensional 
characteristics of the MFCC corresponding to the wall decoration friction sound, the drilling machine working sound and the sound in the elevator compartment under the vertical starting condition. Each point in the figure represents the MFCC feature vectors of each frame sample. All the feature vectors are calculated based on the following parameters: frame length $50 \mathrm{~ms}$, neighbor window overlap length $25 \mathrm{~ms}$, number of triangular filter banks $L$ 41, minimum frequency $20 \mathrm{~Hz}$, maximum frequency $10 \mathrm{kHz}, \mathbf{c}_{\text {mel }}$ length 3 . As is seen in the figure, the three sound samples are obviously differentiated from each other, which indicates that the MFCC features have the ideal clustering analysis potential.

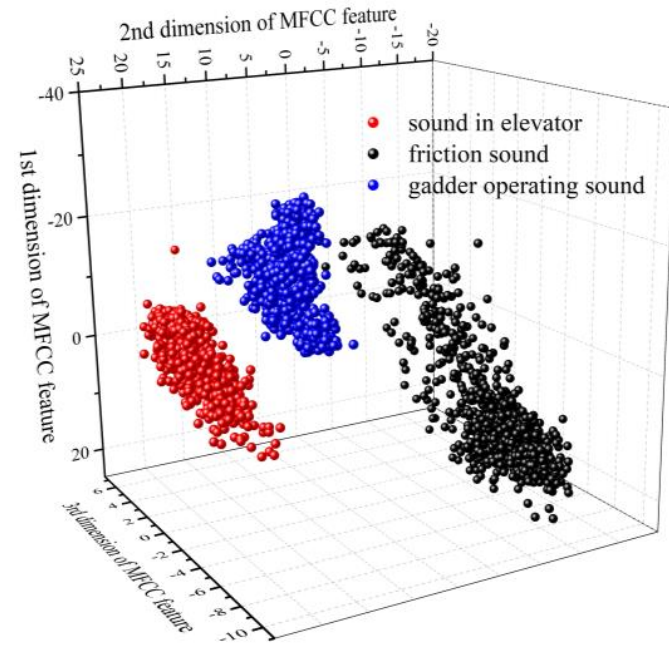

Fig. 6. First 3 dimensions of MFCC features of different sounds

\section{Numerical Test}

In this paper, the sound measured in the construction site of super high-rise building project of Hangzhou Century Center is used as a sample to carry out numerical experiments which are mainly carried out for two aspects: (1) based on the established de-noising method, the effects of noise on the spectral centroid and MFCC features of the pipeline sound signal are investigated. (2) the effectiveness of the proposed scheme is verified by the simulation of the synthesized pipeline sound signals in different states.

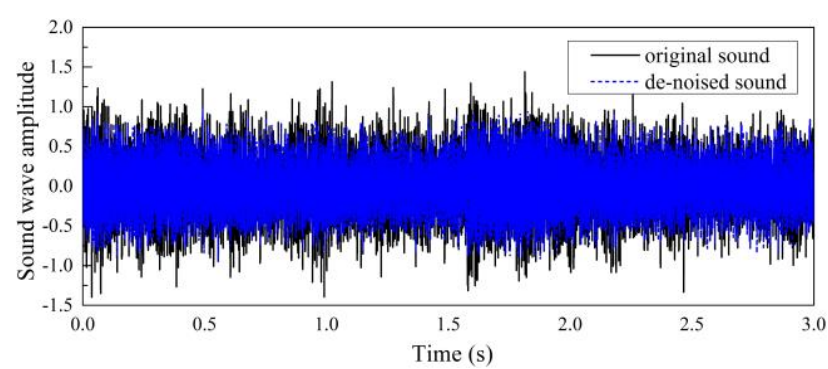

Fig. 7. Sound wave of pumping pipe before and after de-noising
According to the sound acquisition scheme shown in figure 2 , the same type of sound acquisition devices, mic 1 , mic 2 , mic 3 and mic 4 , are installed at the distance of $r_{2}=2.0 \mathrm{~m}, r_{3}=4.5 \mathrm{~m}$ and $r_{4}=6.5 \mathrm{~m}$, respectively, adjacent to and around the pipeline, which are used to collect the live sound at the sampling rate of $11.025 \mathrm{kHz}$. Figure 7 shows a 3 -second audio waveform recorded by mic 1 and marked as "original sound". After it is processed by the de-noising algorithm proposed by the present paper, the sound waveform segment labeled " de-noised sound" is obtained. As you can see, the acoustic signals from the immediate vicinity of the pipeline are mixed with significant noise components. After artificial hearing screening, the algorithm has at least succeeded in filtering the noise identified by the human ear. Readers who are interested may download and listen to the audio files in this article via: https://app.yinxiang.com/shard/s47

/nl/29346280/b90dec1c-8506-4d44-852e-020530013ff7

To examine the effects of noise on sound features, Figures 8 and 9 show the short-time spectral centroids and the first two-dimensional MFCC feature vectors before and after de-noising respectively. In the calculation of spectrum centroids, the short frame length is $20 \mathrm{~ms}$ and the overlap length of adjacent window is 10 $\mathrm{ms}$. In the calculation of MFCC vectors, the short frame length is $20 \mathrm{~ms}$ and the overlap length of adjacent window is $10 \mathrm{~ms}$, the number of triangular filters $L$ is 41 , the lowest frequency is $20 \mathrm{~Hz}$, the highest frequency is 5 $\mathrm{kHz}$ and the length of $\mathbf{c}_{\text {mel }}$ is 2 . Figure 8 shows that the noise in the pipeline generally reduces the centroid effects of the spectrum; Figure 9 shows that the noise components generally reduce the first-dimension coefficient of the MFCC feature vectors and increase the 2nd dimension coefficient of the MFCC feature vectors. Before and after de-noising, the feature center of MFCC is shifted from [28.760, 4.751] to [25.592, 5.609].

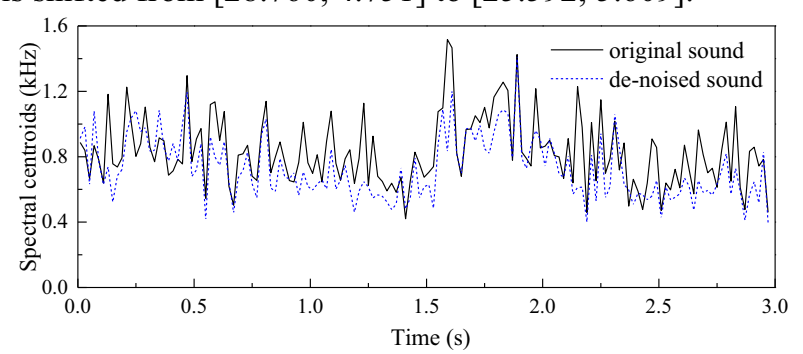

Fig. 8. Spectral centroids of pipeline before/after de-noising

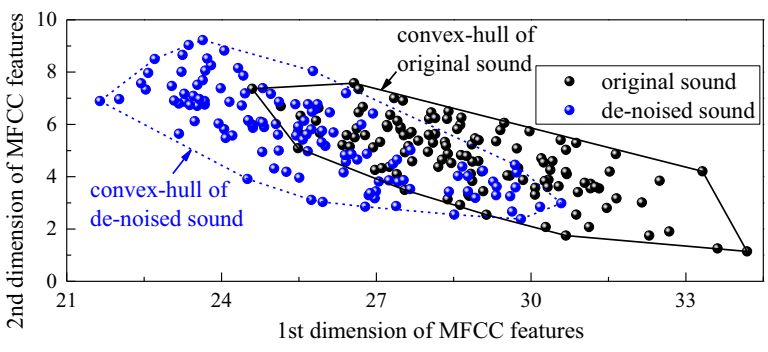

Fig. 9. MFCC features of pipeline before/after de-noising

\subsection{Effect of Noise on Features}

\subsection{Sound clustering}


In order to test the validity of the sound monitoring and recognition scheme, this paper simulates the possible sound signals of the pipeline by synthesizing the sound of the pipeline in different working states, and tests the sound recognition program of the pipeline based on it. In the manual simulation process, the sound samples collected in the field for the smooth transportation of concrete by the 40 -second pipeline are considered as "normal", and the sound samples collected from the pipeline in the pressurization stage of concrete pumping equipment are considered as "abnormal". In addition, the simulation synthesis method considers the continuity of the sound state changes in the concrete pump pipe operation. Figure 10 shows the de-noised sound waveform, where the first $0 \sim 40$ second samples falls in the "normal" category while the samples of $40 \sim 53$ seconds falls in the stage of pressurization. Signals after 53 seconds falls in the high-pressure state.

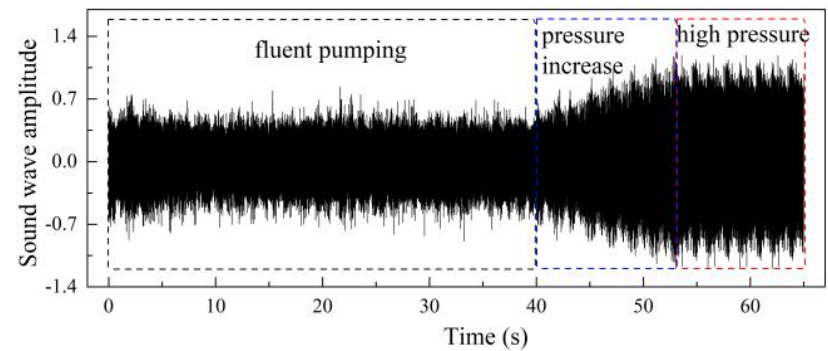

Fig. 10. Manual pipeline sounds at different stages

The first start-up of the pipeline sound recognition system usually assumes that the sound signals are in the category of "normal" at the initial stage. Therefore, this example is carried out to demonstrate the algorithm, and it is assumed that the first 20 seconds of the sound samples are in the "normal" category. The program automatically determines the distance between the signal feature and the convex hull of the "normal" category after 20 seconds.

In order to reduce the length of the short-time feature vectors of the sound signal without losing the sound state distinguishing ability of the feature vectors, Figure 11 previews the change of MFCC feature vectors of the sound samples. As is seen in the figure, the first dimension of MFCC vectors are significantly different from the other dimensions. Therefore, the center of mass of the short-time spectrum is used as the first dimension of the short-time feature vectors of the sound signal while the first dimension and the second dimension of MFCC vectors are used as the second dimension and the third dimension of short-time feature vectors of sound signals. In addition, the "anomaly" threshold of the sound is set as 3.75. Figure 12 shows the distance between the feature points of the sound signals after 20 seconds and the convex hull formed by the set of feature points in the first 20 seconds. The distance curve shows that the distance fluctuates violently with time and it is not convenient for the program to judge the sound state stably. Therefore, this paper adopts Gauss filtering algorithm and uses 100 data points as the window width to process the weighted average, which helps to get the smooth curve shown by the dotted line in figure. Figure 12 shows that the pipeline sound is in the "normal" state during 20 to 44.38 seconds. The pipeline is in the changing state during 44.38 to 51.50 seconds, which is used as the basis of the sound recognition system for the early warning of the pipeline installation state. After 51.50 seconds, the pipeline is in a stable "abnormal" state. Manually verified, if the "abnormal" state is not the one of safety accident, the new clustering and its convex hull can be formed by sound signals after 51.5 seconds, which is convenient to calculate the distance between the new feature point and the convex hull when the signal changes.

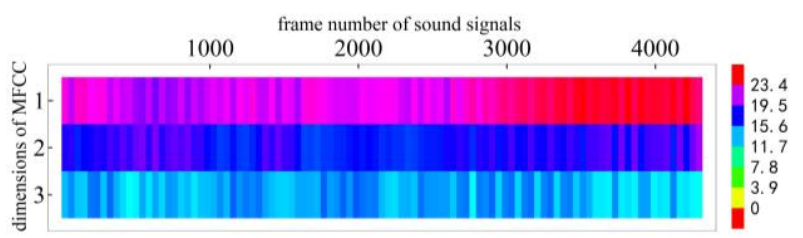

Fig. 11. Transition of the MFCC of sound sample

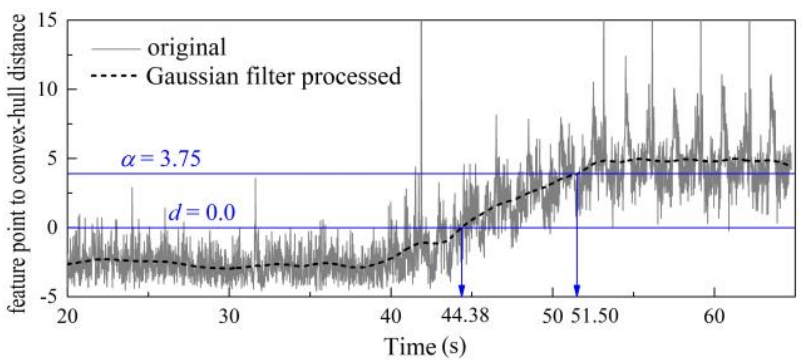

Fig. 12. Distance from feature points to convex hull of normal cluster after 20 seconds

\section{Conclusion}

Based on the characteristics of sound propagation, a noise filtering algorithm for construction site environment is proposed in this paper. The method of sound state clustering based on the short-time spectrum centroids and MFCCs is proposed where the distance from feature vector to cluster convex hull is used as the clustering basis. On the one hand, the numerical test verifies the effectiveness and necessity of the proposed noise reduction algorithm. On the other hand, the sound signal clustering and recognition algorithm are used to simulate the safety monitoring and early warning process of the pipeline in the construction site. The numerical results show that:

(1) the effect of high-decibel noise on the real signal characteristic coefficient of the pipeline cannot be ignored;

(2) the MFCC feature vectors with fewer dimensions can be used to characterize the sound of pipeline in construction site. In the case mentioned in the paper, the feature vectors composed of the MFCCs with length no greater than 4 can be used to classify the sound state of pipeline;

(3) the safety monitoring method of the pipeline by sound clustering this paper proposes, is capable of monitoring and early warning of the state of the pipeline. 


\section{Acknowledgments}

The author wish to acknowledge the support from National Key R \& D Program of China (Grant No. 2017YFC0805500) and Research Fund of Shanghai Construction Group (Grant No. 19YJKF-19)

\section{References}

1. Secrieru E, Mohamed W, Fataei S, et al. (2020) Assessment and prediction of concrete flow and pumping pressure in pipeline. Cem. Concr. Compos., 107: 1-13.

2. Ngo T-T, Kadri E-H, Cussigh F, et al. (2011) Measurement and modeling of fresh concrete viscous constant to predict pumping pressures. Can. J. Civ. Eng., 38: 944-956.

3. Wu B, Chen B, Xu J, et al. (2011) Analysis on pumping pressure loss of high-strength and high-performance concrete. Concrete, 33: 142-144. (in Chinese)

4. Kwon SH, Jang KP, Kim JH, et al. (2016) State of the art on prediction of concrete pumping. Int. J. Concr. Struct. Mater., 10: S75-S85.

5. Kaplan D, Larrard Fd, Sedran T. (2005) Design of concrete pumping circuit. ACI Mater. J., 102: $110-117$.

6. Feys D, Khayat KH, Perez-Schell A, et al. (2015) Prediction of pumping pressure by means of new tribometer for highly-workable concrete. Cem. Concr. Compos., 57: 102-115.

7. Chen J, Xie H, Guo J, et al. (2019) Preliminarily experimental research on local pressure loss of fresh concrete during pumping. Measurement, 147: $1-9$.

8. Mai C-T, Kadri E-H, Ngo T-T, et al. (2014) Estimation of the pumping pressure from concrete composition based on the identified tribological parameters. Adv. Mater. Sci. Eng., 2014: 1-18.
9. Chen M, Xu Z. (2009) Study on damage detection of concrete based on ultrasonic pulse compression method. China Safty Sci. J., 19: 100-104. (in Chinese)

10. Xie X, Yi W, Wang X, et al. (2008) Review of structural damage detecting based on dynamics system identification in time domain. China Safty Sci. J., 18: 110-115.(in Chinese)

11. Trtnik G, Kavcic F, Turk G. (2009) Prediction of concrete strength using ultrasonic pulse velocity and artificial neural networks. Ultrasonics, 49: 53-60.

12. He W. (2019) Mechanical signal recognizng based on semi-supervised manifold learning. Sh. Electron. Eng., 39: 207-212.(in Chinese)

13. Anastasopoulos A, Kourousis D, Bollas K. (2009) Acoustic emission leak detection of liquid filled buried pipeline. J. Acoustic Emission. 27: 27-39

14. Theodoridis S, Koutroumbas K. (2009) Pattern recognition, fourth edition. Elsevier, Singapore.

15. Zhao H, Zhang L, Chen Z. (2009) Using mixed window function and subband spectrum centroid in MFCC feature extractmn process. J. Comput. Appl., 29: 49-51.(in Chinese)

16. Li H. (2019) Statistical learning method. Tsinghua University Press, Beijing.(in Chinese)

17. O'Rourke J. (1998) Computational geometry in C, 2nd edition. Cambridge University Press, England.

18. Wang N, Chen K. (2009) Application of sub-band spectral centroid features to recognizing underwater targets. Acta Armamentarii, 30: 144-149.(in Chinese) 